\title{
Optimal treatment strategy for type A acute aortic dissection with intramural hematoma
}

\author{
Mitsumasa Hata, MD, PhD, Hiroaki Hata, MD, Akira Sezai, MD, Isamu Yoshitake, MD, \\ Shinji Wakui, MD, and Motomi Shiono, MD
}

\begin{abstract}
Objective: The management strategy for type A acute aortic dissection (AAD) with intramural hematoma (IMH) remains controversial. The aim of this study was to compare the outcomes of emergency surgery and medical treatment for patients with acute type A IMH.
\end{abstract}

\begin{abstract}
Methods: One hundred seventy-one patients with acute type A IMH, whose average age was 69.4 years (range, 39-90 years) were divided into 3 groups: groups I and II consisted of 74 and 33 patients undergoing emergency surgery for complicated and uncomplicated type A IMH, respectively, and group III comprised 66 patients who were treated conservatively. We compared the 3 groups in terms of mortality and morbidity.
\end{abstract}

Results: In group I, postoperative hospital mortality was 5.4\% (4 patients). In group II, all patients survived without any complications after surgery. On the other hand, in group III, 5 patients underwent emergency surgery within 7 days after onset owing to newly developed cardiac tamponade and $17(25.8 \%)$ patients died in the hospital, which was a significantly higher rate than those in groups I and II. Furthermore, although the group III patients survived, 5 patients required mechanical ventilation owing to heart failure or pneumonia, and 4 patients had a stroke during the hospital stay. There was no difference in actuarial survivals at 10 years, which were $64.2 \%$ in group I, $64.9 \%$ in group II, and $68.7 \%$ in group III.

Conclusions: Emergency surgery for patients with type A IMH showed favorable mortality rates because most of the patients were in hemodynamically stable condition preoperatively. On the other hand, several patients died suddenly during medical care. Emergency surgical treatment may provide a better outcome than medical treatment at the time of onset, even for patients with type A IMH. (J Thorac Cardiovasc Surg 2014;147:307-11)

With the development of computed tomography (CT) and magnetic resonance imaging, which confirmed that clots and hematomas fill false lumina, steps in the progression of hematomas have become clear. This type of dissection is widely known as thrombosed dissection or intramural hematoma (IMH). Much of the controversy originates from inherent limitations of previous studies to assess whether surgery or conservative treatment should be performed for type A acute aortic dissection (AAD) with $\mathrm{IMH}^{1,2}{ }^{1,2}$ In Eastern countries, there has been a growing trend to treat type A IMH nonoperatively, ${ }^{2}$ whereas surgery has long been accepted in the West. ${ }^{1,3}$ Even if the initial CT scan reveals an IMH, emergency surgical treatment is occasionally required in as much as cardiac tamponade could develop during the hospital stay. However, information comparing surgical intervention with nonsurgical care of patients with type A IMH is

\footnotetext{
From the Department of Cardiovascular Surgery, Nihon University School of Medicine, Tokyo, Japan.

Disclosures: Authors have nothing to disclose with regard to commercial support. Received for publication June 27, 2012; revisions received Sept 30, 2012; accepted for publication Nov 6, 2012; available ahead of print Dec 10, 2012.

Address for reprints: Mitsumasa Hata, MD, PhD, Department of Cardiovascular

Surgery, Nihon University School of Medicine, 30-1 Ooyaguchi Kamimachi

Itabashi-ku, Tokyo 173-8610, Japan (E-mail: hata.mitsumasa@nihon-u.ac.jp). $0022-5223 / \$ 36.00$

Copyright $(2014$ by The American Association for Thoracic Surgery

http://dx.doi.org/10.1016/j.jtcvs.2012.11.015
}

limited. The aim of this study was to compare the outcomes of emergency surgery and medical treatment for patients with acute type A IMH.

\section{PATIENTS AND METHODS}

Between December 1998 and March 2012, 388 patients with AAD were admitted to our institution. Initial CT scans revealed an IMH in 171 $(44.1 \%)$ patients. Institutional review board approval was provided before publication of this article and reporting of the information. All patients were admitted to the intensive care unit. One hundred three $(60.2 \%)$ patients were female, and the average age was $69.4 \pm 11.1$ years, ranging from 39 to 90 years. All patients had a history of hypertension. There were no patients with Marfan syndrome. Contrast CT was performed on all patients as soon as they were referred to the hospital. Transthoracic echocardiography was then performed to detect pericardial effusion and assess aortic valve regurgitation (AR) and cardiac function. We obviously selected emergency surgical treatment for the patients with type A IMH complicated by cardiac tamponade, AR, acute myocardial infarction (AMI), or other vital organ malperfusion. However, even when the patients were not in a state of shock owing to such critical complications, we performed emergency surgery if pericardial effusion or hematoma was found in the initial CT or echocardiogram, regardless of the maximum aortic diameter. Patients were divided into 3 groups: group I consisted of 74 patients undergoing emergency surgery for complicated IMH such as tamponade, AR, AMI, or vital organ malperfusion; group II comprised 31 hemodynamically stable patients undergoing emergency surgery for uncomplicated IMH; and group III comprised 66 patients who were treated conservatively, first with intravenous administration of calcium antagonist, beta-blocker and pain killer to maintain systolic blood pressure at less than $120 \mathrm{~mm}$ $\mathrm{Hg}$ for 2 days, and then with these medications in the form of oral tablets. In group I, $62(83.8 \%)$ patients were in a preshock state owing to cardiac 


\section{Abbreviations and Acronyms \\ $\mathrm{AAD}=$ acute aortic dissection \\ $\mathrm{AMI}=$ acute myocardial infarction \\ $\mathrm{AR}=$ aortic valve regurgitation \\ $\mathrm{CT}=$ computed tomography \\ $\mathrm{IMH}=$ intramural hematoma}

tamponade. The incidences of mild to moderate AR, AMI, and lower body ischemia were $6(8.1 \%), 4(5.4 \%)$, and $2(2.7 \%)$ patients, respectively. Six $(8.1 \%)$ patients required intubation before arriving at the hospital and underwent cardiopulmonary resuscitation administered by an electro-defibrillator before entering the operating theater. In group II, all patients were in hemodynamically stable condition despite hemopericardium. On the other hand, none of the patients in group III had critical complications and all were in hemodynamically stable condition at the time of admission. We kept the medically treated patients in the intensive care unit for 7 days even though no complications such as pneumonia or heart failure occurred. In groups I and II, the patients took a walk and started early exercise training on postoperative day 3 . We compared the 3 groups in terms of in-hospital mortality, morbidity, and long-term outcome.

\section{Surgical Procedure}

Cardiopulmonary bypass was implemented through femoral arterial cannulation. A 2-stage venous cannula was inserted into the right atrium, except in patients with preoperative shock $(<60 \mathrm{~mm} \mathrm{Hg}$ of systolic blood pressure). If a patient had evidence of shock owing to cardiac tamponade, femorofemoral circulatory assistance was initiated before the chest was opened. Deep hypothermic circulatory arrest and antegrade selective cerebral perfusion were used for cerebral protection until December 2005. Each patient was cooled to $20^{\circ} \mathrm{C}$ (rectal temperature). Since January 2006 we have used mild hypothermic arrest $\left(28^{\circ} \mathrm{C}\right)$ without cerebral perfusion. Tear-oriented replacement of the aortic segment containing the intimal tear was used as much as possible. However, if the intimal tear was located in the distal arch to descending aorta, only the ascending aorta was replaced.

The mean duration of follow-up was 62.6 months, ranging from 1 to 185 months. Follow-up information was obtained for all patients because we looked after the patients in our outpatient clinic. For parametric data, the results are expressed as the mean \pm standard deviation. Statistical calculations were undertaken with StatView (SAS Institute, Inc, Cary, NC). Statistically significant differences were determined from parametric and nonparametric data using the analysis of variance and $\chi^{2}$ test, respectively. Independent predictor for early event in group III was examined using the univariate analysis with Fisher's exact test and subsequent multivariate logistic regression analysis from 9 parameters, including age $(>80$ or $<65$ years), sex, maximum aortic diameter $(>50 \mathrm{~mm})$, and thickness of false lumen $(>10 \mathrm{~mm})$ at the time of onset, prevalence of chronic renal failure, and diabetes. The actuarial survival was calculated using the Kaplan-Meier method.

\section{RESULTS}

There were no differences between the groups in terms of age, sex, maximum ascending aortic diameter, thickness of $\mathrm{IMH}$, case with aortic diameter of more than $50 \mathrm{~mm}$, history of stroke, chronic renal failure, or diabetes (Table 1). In groups I and II, 100 (90.9\%) patients underwent ascending to proximal hemiarch replacement. Total arch replacement was required in $10(9.1 \%)$ patients. Coronary bypass was concomitantly carried out in 2 patients. Intimal tears were resected in $71(64.5 \%)$ patients. In group I, $5(6.8 \%)$ patients were taken back to the operating theater owing to bleeding (Table 2). The incidences of postoperative stroke, pneumonia, and renal failure were $4(5.4 \%), 2(2.7 \%)$, and $1(1.4 \%)$ patient, respectively. Postoperative hospital mortality rate was $5.4 \%$ (4 patients) (Table 2 ). In group II, all patients survived without any complications. In group III, 5 patients had a tamponade state newly develop within 7 days after onset despite the fact that the initial CT scan did not show any hematoma in the pericardium. These patients underwent emergency surgery but 2 of them died of multiple organ failure. Furthermore, $15(22.7 \%)$ patients in group III died in the hospital of cardiac tamponade (9 patients), ischemic colitis (2 patients), stroke (1 patient), subarachnoid hemorrhage (1 patient), AMI (1 patient), and congestive heart failure (1 patient). Finally, hospital mortality rate was $25.8 \%$ (17 patients) in group III. This number was significantly higher than that in groups I and II $(P=.0010)$ (Table 2$)$. In group III, although the patients survived, 5 patients required mechanical ventilation owing to heart failure or pneumonia and 4 patients had a stroke during the hospital stay (Table 2). Univariate analysis revealed that female gender $(P=.0267)$ and false lumen thickness of more than $10 \mathrm{~mm}(P=.0415)$ were associated with newly developed cardiac tamponade in group III. However, multivariate logistic regression analysis failed to reach statistical significance. Average hospital stay was significantly shorter in groups I and II than in group III $(P=.0024$, Table 2$)$. In groups I and II, most of the patients were discharged from the hospital early after the operation and went to an outpatient cardiac rehabilitation center. In group III, however, most of the patients required a longer stay in our hospital and followed by our outpatient clinic after discharge from the hospital. In group III, 20 patients underwent elective surgery because of dilation $(>50 \mathrm{~mm})$ of the ascending aorta within 12 months after onset. In the later phase, 8 group I patients died of AMI, pneumonia, malignancy, stroke, or late-stage senility and 3 group II patients died of abdominal aortic rupture, traffic accident, or heart failure. On the other hand, in group III, 6 patients died of cerebral hemorrhage, pneumonia, stroke, or rupture of the distal aortic arch in the later stage. There was no difference in actuarial survivals at 10 years, which were $64.2 \%$ in group I, $64.9 \%$ in group II, and $68.7 \%$ in group III (Figure 1). Freedom from ascending aortic event including 5 emergencies, early death owing to newly developed cardiac tamponade, and late surgery in group III was $46.8 \%$ at 10 years (Figure 2).

\section{DISCUSSION}

The efficacy of emergency surgical intervention for classic AAD with a patent false lumen is widely accepted, but its role in the treatment of the patients with IMH 
TABLE 1. Patient profile

\begin{tabular}{lcccc}
\hline & & & & $\boldsymbol{P}$ \\
& Group I & Group II & Group III & value \\
\hline Age (y) & $70.1 \pm 12.0$ & $68.1 \pm 11.8$ & $69.2 \pm 9.6$ & NS \\
Sex (male/female) & $31 / 43$ & $14 / 31$ & $46 / 66$ & NS \\
Maximum diameter (mm) & $51.0 \pm 465$ & $50.9 \pm 4.3$ & $50.1 \pm 4.1$ & NS \\
Thickness of IMH (mm) & $12.2 \pm 1.6$ & $12.0 \pm 1.8$ & $11.6 \pm 1.6$ & NS \\
Case of $>50 \mathrm{~mm}$ & $44(59.5 \%)$ & $18(58.1 \%)$ & $33(50 \%)$ & NS \\
Diabetes & $11(14.9 \%)$ & $4(12.9 \%)$ & $8(12.1 \%)$ & NS \\
Stroke (patients) & $4(5.4 \%)$ & $1(3.2 \%)$ & $2(3.0 \%)$ & NS \\
CRF (patients) & $2(2.7 \%)$ & $1(3.2 \%)$ & $3(4.5 \%)$ & NS \\
\hline
\end{tabular}

$N S$, Not significant; $I M H$, intramural hematoma; $C R F$, chronic renal failure.

remains unclear. Several investigators reported that aggressive medical treatment provided complete resorption of the thrombosed false lumen and a favorable hospital course. ${ }^{4,5}$ Song and colleagues ${ }^{6}$ recommended that initial medical treatment followed by timed surgery in patients with complications be practiced for stable patients with type A IMH. However, surgical outcomes for redissection from IMH may be poor because patients suddenly become very sick and hemodynamically unstable at the time of surgery. ${ }^{7}$ Therefore, controversy still exists as to whether emergency surgical intervention should be carried out for the patients with type A IMH. Apart from the international heterogeneity in the relative prevalence of type A $\mathrm{IMH}$, geographic differences in the clinical outcomes of medical treatment and the role of emergency surgery remain challenging issues. ${ }^{8}$ Some European investigators have reported poor outcomes with medical treatment alone and advocated emergency surgery, ${ }^{1,9}$ whereas other studies in Japan and Korea showed comparable outcomes in the absence of emergency surgery. ${ }^{2,4,5}$ However, these studies were not randomized trials, and numbers of enrolled patients were fewer than 40 even in multicenter studies; these numbers are certainly too low to offer the required statistical power. In the present study, the subject consisted of more than 170 consecutive patients. Therefore, we are confident about the results from the statistical assessment despite this being a single-center trial.

TABLE 2. Hospital mortality and morbidity

\begin{tabular}{lcccc}
\hline & & & & $\begin{array}{c}\boldsymbol{P} \\
\text { value }\end{array}$ \\
\hline Reexploration (patients) & $5(6.8 \%)$ & 0 & & \\
Stroke (patients) & $4(5.4 \%)$ & 0 & $4(6.1 \%)$ & NS \\
Pneumonia (patients) & $2(2.7 \%)$ & 0 & $2(3.0 \%)$ & NS \\
Heart failure (patients) & 0 & 0 & $3(4.5 \%)$ & NS \\
Renal failure (patients) & $1(1.4 \%)$ & 0 & $1(1.5 \%)$ & NS \\
Hospital stay (d) & $16.0 \pm 11.9$ & $15.7 \pm 11.4$ & $26.7 \pm 12.3$ & .0024 \\
Hospital mortality & $4(5.4 \%)$ & 0 & $17(25.8 \%)$ & .001 \\
$\quad$ (patients) & & & & \\
\hline$N S$, Not significant. & & & &
\end{tabular}

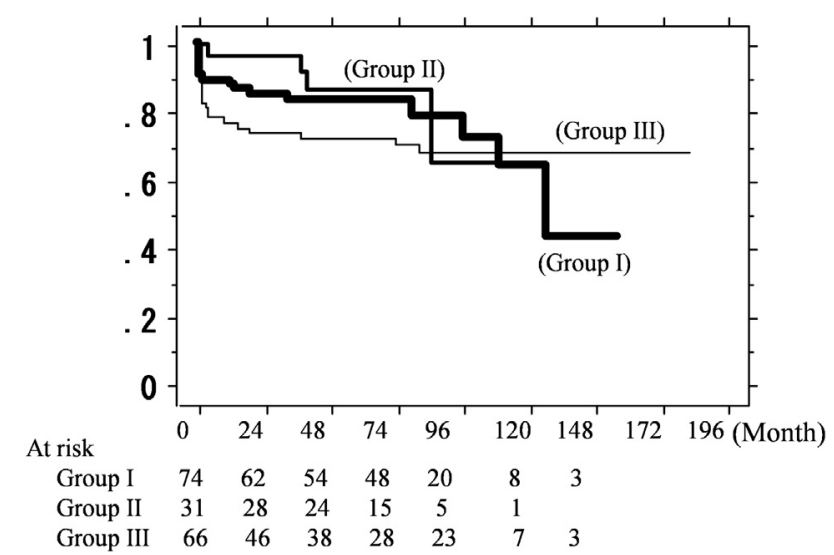

FIGURE 1. Actuarial survival curves. There was no difference in actuarial survivals at 10 years, which were $63.4 \%$ in group I and $68.7 \%$ in group II $(P=.128)$.

In the present study, we obviously selected emergency surgical treatment for the patients with shock state owing to critical complications. Furthermore, emergency surgery was also recommended as soon as possible for group II patients who showed evidence of slight hemopericardium in the initial CT scan, even if the patients were in a stable condition. In these cases, the patients were gradually becoming hypotensive before entering the operating theater, and approximately $500 \mathrm{~mL}$ of blood and hematoma were actually presented at the time of surgery. Even though CT or echocardiographic scans on admission did not show any pericardial effusion, blood oozing may have developed gradually. Sudden death is the most tragic event associated with type A $\mathrm{IMH}$, and the most probable reason for sudden death is aortic rupture. Therefore, group III included the 5 patients who suddenly required emergency surgery because of the development of a preshock state owing to cardiac tamponade. Furthermore, a shock state suddenly developed in the other 9 patients and they could not reach the operating theater even though the initial CT scan did not show any hematoma

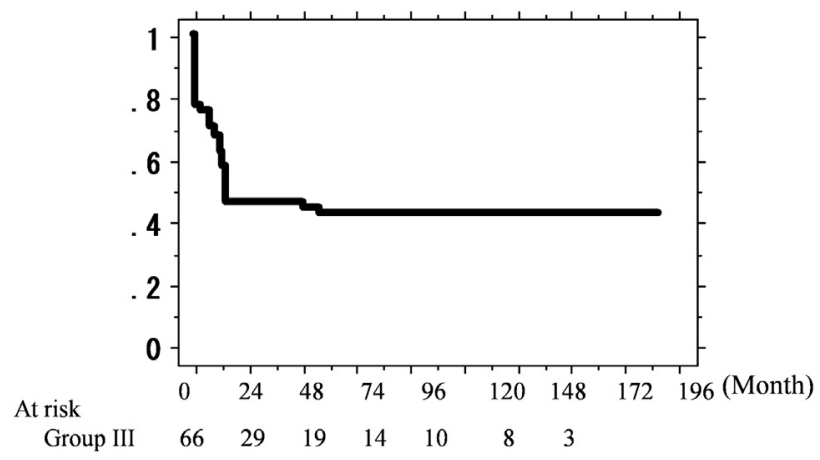

FIGURE 2. Freedom from aortic event in group III. Freedom from ascending aortic event including 5 emergencies, early death owing to newly developed cardiac tamponade, and late surgery in group III was $46.8 \%$ at 10 years. 
into the pericardium. Early investigators already reported that aortic rupture occurred much more commonly in patients with type A IMH than in those with AAD with a patent false lumen, resulting in high mortality. ${ }^{10}$ Therefore, even when an initial CT scan reveals a type A IMH, performing surgery as soon as possible is considered to be a key factor of success.

In group III, 9 patients suddenly died of newly developed cardiac tamponade during the hospital stay. Finally, 15 patients died of critical complications during the early stage after onset. Furthermore, even though the patients survived, 5 patients had complications of pneumonia and heart failure and required mechanical ventilators. Even though the patients survived, releasing the patient's mental and physical stress may be difficult using bed rest management. Of course, bed rest is necessary and important for antihypertensive therapy, particularly in critically ill patients. However, it is associated with risk factors of pneumonia, heart failure, and depression. ${ }^{11}$ Therefore, it is considered that conservative medical management may be associated with early cardiopulmonary complications for patients with type A IMH. On the other hand, in surgical treatment groups, the patients could eat and drink on the first postoperative day. Furthermore, they could take a walk and start early exercise training on postoperative day 3 because the dissecting cavity had already been repaired. In groups I and II, most of the patients could go home approximately 15 days after surgery and visit the cardiac rehabilitation center by themselves. Therefore, emergency surgical treatment should enable much easier management of patients and reduced incidence of critical complications compared with medical management during the early phase after onset of acute type A IMH.

In the present study, 4 (5.4\%) patients in group I died after surgery. This hospital mortality rate was remarkably low compared with previous studies ${ }^{12,13}$ of patients with AAD. In particular, all group II patients survived without any complications. In groups I and II, all patients underwent emergency surgery within 24 hours of onset. Earlier referral to the operating theater has been improving surgical results before dissection-related complications become irreversible. ${ }^{14}$ In fact, 2 of 5 group III patients who were converted to emergency surgery owing to newly developed cardiac tamponade within 7 days after onset died of multiple-organ failure after surgery. At that time, the patients already had complications of renal failure and severe coagulopathy before surgery. Makino and colleagues ${ }^{7}$ also reported that surgical outcomes for the patients with type A IMH converted from medical treatment are poor because patients suddenly become very sick and hemodynamically unstable at the time of surgery. Furthermore, 9 of 15 group III hospital deaths occurred within 7 days after onset owing to newly developed cardiac tamponade. It was too late to prepare the operating theater for the patients. Although univariate analysis showed that female gender and false lumen thickness were associated with newly developed cardiac tamponade, the series were too small to allow a meaningful multivariate analysis. Song and colleagues ${ }^{6}$ also recommend early surgical treatment for patients with false lumen thickness of more than $11 \mathrm{~mm}$. In the present study, however, both the maximum aortic diameter and false lumen thickness were similar between the groups. Current available surgical techniques yield good immediate and longterm results with minimal risk in surgery for AAD. ${ }^{14}$ As shown by the results in our group II patients, we believe that most of patients with uncomplicated type A IMH can be rescued by emergency surgical treatment. On the other hand, most patients requiring cardiopulmonary resuscitation could not reach even the operating theater. ${ }^{15,16}$ In cases in which there had been uncertainly about whether to go ahead with surgery, the patients died suddenly. Therefore, even if the patient has type A IMH and is in stable condition at the time of onset, emergency surgery should be recommended.

\section{CONCLUSIONS}

Emergency surgery for patients with type A IMH showed favorable mortality rates because most of the patients were hemodynamically stable preoperatively. On the other hand, several patients died suddenly during medical care. Emergency surgical treatment may provide a better outcome than medical treatment at the time of onset, even for patients with type A IMH.

\section{References}

1. Nienaber CA, von Kodolitsch Y, Petersen B, Loose R, Helmchen U, Haverich A, et al. Intramural hemorrhage of the thoracic aorta. Diagnostic and therapeutic implications. Circulation. 1995;92:1465-72.

2. Kaji S, Akasaka T, Horibata Y, Nishigami K, Shono H, Katayama M, et al. Longterm prognosis of patients with type A aortic intramural hematoma. Circulation. 2002;106(12 Suppl 1):I248-52.

3. Harris KM, Braverman AC, Gutierrez FR, Barzilai B, Davila Roman VG. Transesophageal echocardiographic and clinical feature of aortic intramural hematoma. J Thorac Cardiovasc Surg. 1997;114:619-26.

4. Song JK, Kim HS, Kang DH, Lim TH, Song MG, Park SW, et al. Different clinical features of aortic intramural hematoma versus dissection involving the ascending aorta. J Am Coll Cardiol. 2001;37:1604-10.

5. Moizumi Y, Komatsu T, Motoyoshi N, Tabayashi K. Management of patients with intramural hematoma involving the ascending aorta. J Thorac Cardiovasc Surg. 2002;124:918-24

6. Song JK, Yim JH, Ahn JM, Kim DH, Kang JW, Lee TY, et al. Outcomes of patients with acute type A aortic intramural hematoma. Circulation. 2009;120: 2046-52.

7. Makino Y, Sato K, Sugiki T, Murakami T. Treatment strategy for Stanford type A acute aortic dissection with a thrombosed false lumen. Jpn J Vasc Surg. 2012;21: 11-3.

8. Pelzel JM, Braverman AC, Hirsch AT, Harris KM. International heterogeneity in diagnostic frequency and clinical outcomes of ascending aortic intramural hematoma. J Am Soc Echocardiogr. 2007;20:1260-8.

9. Evangelista A, Dominguez R, Sebastia C, Salas A, Permanyer-Miralda G, Avegliano G, et al. Prognostic value of clinical and morphologic findings in short-term evolution of aortic intramural hematoma: therapeutic implications. Eur Heart J. 2004;25:81-7.

10. Coady MA, Rizzo JA, Elefteriades JA. Pathologic variants of thoracic aortic dissections: penetrating atherosclerotic ulcers and intramural hematoma. Cardiol Clin. 1999; 17:637-57. 
11. Niino T, Hata M, Sezai A, Yoshitake I, Unosawa S, Shimura K, et al. Optimal clinical pathway for the patient with type B acute aortic dissection. Circ J. 2009; 73:264-8.

12. Mehta RH, Suzuki T, Hagan PG, Bossone E, Gilon D, Llovet A, et al. Predicting death in patients with acute type a aortic dissection. Circulation. 2002;105: 200-6.

13. Mehta RH, O'Gara PT, Bossone E, Nienaber CA, Myrmel T, Cooper JV, et al. Acute type A aortic dissection in the elderly: clinical characteristics, management, and outcomes in the current era. J Am Coll Cardiol. 2002;40:685-92.
14. Hata M, Sezai A, Yoshitake I, Wakui S, Minami K, Shiono M. Midterm outcome of rapid, minimally invasive resection of acute type A aortic dissection. Ann Thorac Surg. 2010;89:1860-5.

15. Shiono M, Hata M, Sezai A, Iida M, Yagi S, Negishi N. Emergency surgery for acute type A aortic dissection in octogenarians. Ann Thorac Surg. 2006;82:554-9.

16. Hata M, Shiono M, Inoue T, Sezai A, Niino T, Funahashi M, et al. Preoperative cardiopulmonary resuscitation is the only predictor for operative mortality of type A acute aortic dissection: a recent 8-year experience. Ann Thorac Cardiovasc Surg. 2004;10:101-5. 